\title{
Comparação da eficácia anestésica entre infiltração bucal com articaína $4 \%$ e bloqueio do nervo alveolar inferior com lidocaína 2\%: um ensaio clínico prospectivo, randomizado e duplo cego
}

\author{
Comparison of anesthetic efficacy between oral infiltration with $4 \%$ articaine and inferior alveolar \\ nerve block with $2 \%$ lidocaine: a prospective, randomized, double-blind clinical trial \\ Comparación de la eficacia anestésica entre la infiltración oral con articaína al $4 \%$ y el bloqueo del \\ nervio alveolar inferior con lidocaína al 2\%: ensayo clínico prospectivo, aleatorizado y doble ciego
}

\section{Resumo}

Para analgesia de dentes inferiores posteriores, a técnica anestésica mais utilizada é o bloqueio dos nervos alveolar inferior e lingual, apesar de seus insucessos clínicos relevantes. O objetivo do presente estudo foi comparar a eficácia anestésica da infiltração supraperiostal com articaína 4\% (INFILT), em comparação ao bloqueio do nervo alveolar inferior com lidocaína 2\% (BNAI), ambos com epinefrina 1:100.000, em dentes posteriores inferiores. Foram selecionados 60 voluntários, de ambos os gêneros, com indicação de restaurações classe I ou II de Black em elementos dentários posteriores mandibulares. O aparelho pulp tester elétrico foi utilizado para avaliação dos seguintes parâmetros: tempo de latência pulpar (tempo decorrido desde o término da injeção até a primeira medida sem sensibilidade), duração do efeito anestésico pulpar (tempo decorrido entre a primeira e última medidas sem sensibilidade) e sucesso anestésico (mínimo de duas medidas consecutivas sem sensibilidade). A dor durante a realização da técnica foi mensurada por meio de uma escala de dor de 11 pontos em caixa. A taxa de sucesso apresentada pela INFILT foi maior que a do BNAI ( $\mathrm{p}=0,0149)$. Por outro lado, não foram encontradas diferenças significativas no que diz respeito a tempo de latência $(\mathrm{p}=0,4755)$, duração do efeito anestésico pulpar $(\mathrm{p}=0,1381)$ e dor à punção e injeção $(\mathrm{p}=0,9256)$. O índice de complicações pós-operatórias foi de $36,7 \%$ em ambos os grupos, sendo todas classificadas como de baixa gravidade, garantindo a segurança clínica para utilização de ambas as técnicas. A infiltração com articaína apresentou maior índice de sucesso em comparação ao bloqueio do nervo alveolar inferior com lidocaína. Uma vez tendo sido alcançada a anestesia local, não houve diferenças na duração do efeito entre ambas as técnicas.

Palavras-chave: Odontologia; Anestesia local; Infiltração dentária; Bloqueio nervoso.

\footnotetext{
Abstract

For analgesia of posterior mandibular teeth, the most used anesthetic technique is the blockade of the inferior alveolar and lingual nerves, despite its relevant clinical failures. The aim of the present study was to compare the anesthetic efficacy of supraperiosteal infiltration with $4 \%$ articaine (INFILT) compared to inferior alveolar nerve block with $2 \%$ lidocaine (BNAI), both with 1:100,000 epinephrine, in lower posterior teeth. Sixty volunteers of both genders were
} 
selected, with indication for Black Class I or II restorations in mandibular posterior dental elements. The electric pulp tester device was used to evaluate the following parameters: pulp onset time (time from the end of the injection to the first non-sensing measurement), duration of the pulp anesthetic effect (time between the first and last non-sensing measurements) and anesthetic success (minimum of two consecutive measurements without sensitivity). Pain during the technique was measured using an 11-point boxed pain scale. The success rate presented by INFILT was higher than that of BNAI ( $\mathrm{p}=0.0149)$. On the other hand, no significant differences were found with regard to latency time $(\mathrm{p}=0.4755)$, duration of pulpal anesthetic effect $(\mathrm{p}=0.1381)$ and pain on puncture and injection $(\mathrm{p}=0.9256)$. The rate of postoperative complications was $36.7 \%$ in both groups, all of which were classified as having low severity, ensuring clinical safety for the use of both techniques. Infiltration with articaine had a higher success rate compared to inferior alveolar nerve block with lidocaine. Once local anesthesia was achieved, there were no differences in the duration of effect between the two techniques.

Keywords: Dentistry; Local anesthesia; Dental leakage; Nerve block.

\section{Resumen}

Para la analgesia de los dientes posteriores mandibulares, la técnica anestésica más utilizada es el bloqueo de los nervios alveolar inferior y lingual, a pesar de sus relevantes fracasos clínicos. El objetivo del presente estudio fue comparar la eficacia anestésica de la infiltración supraperióstica con articaína al 4\% (INFILT) en comparación con el bloqueo del nervio alveolar inferior con lidocaína al 2\% (BNAI), ambos con epinefrina 1: 100.000, en dientes posteriores inferiores. Se seleccionaron 60 voluntarios de ambos sexos, con indicación para restauraciones Black Class I o II en elementos dentales posteriores mandibulares. El dispositivo de prueba de pulpa eléctrica se utilizó para evaluar los siguientes parámetros: tiempo de inicio de la pulpa (tiempo desde el final de la inyección hasta la primera medición sin detección), duración del efecto anestésico pulpar (tiempo entre la primera y la última medición sin detección) y éxito anestésico (mínimo de dos mediciones consecutivas sin sensibilidad). El dolor durante la técnica se midió utilizando una escala de dolor encuadrada de 11 puntos. La tasa de éxito presentada por INFILT fue superior a la de BNAI ( $\mathrm{p}=0,0149)$. Por otro lado, no se encontraron diferencias significativas en cuanto al tiempo de latencia ( $\mathrm{p}$ $=0,4755)$, duración del efecto anestésico pulpar $(\mathrm{p}=0,1381)$ y dolor a la punción e inyección $(\mathrm{p}=0,9256)$. La tasa de complicaciones postoperatorias fue del $36,7 \%$ en ambos grupos, todos ellos clasificados como de baja gravedad, lo que garantiza la seguridad clínica para el uso de ambas técnicas. La infiltración con articaína tuvo una mayor tasa de éxito en comparación con el bloqueo del nervio alveolar inferior con lidocaína. Una vez que se logró la anestesia local, no hubo diferencias en la duración del efecto entre las dos técnicas.

Palabras clave: Odontología; Anestesia local; Filtración dental; Bloqueo nervioso.

\section{Introdução}

O bloqueio do nervo alveolar inferior é a técnica mandibular frequentemente usada para o alcance da anestesia local em dentes posteriores inferiores, embora ela nem sempre resulte em uma anestesia pulpar bem sucedida, pois, mesmo quando técnicas apropriadas são utilizadas, apresenta altos índices de insucesso (Karapinar-kazandag, Tanalp, \& Ersev, 2019; Oleson, Drum, Reader, Nusstein, \& Beck, 2010; Tortamano et al., 2013). É normalmente realizada com lidocaína, agente anestésico do grupo das amidas mais difundida e utilizada para induzir anestesia local (Maljaei, Pourkazemi, Ghanizadeh, \& Ranjbar, 2017).

A infiltração bucal em dentes posteriores mandibulares tem se mostrado vantajosa, visto que reduz o tempo de início da anestesia e diminui o percentual de falhas quando comparadas com a técnica troncular (Kaushik \& Moturi, 2018). Entretanto, sua utilização não deve ser generalizada, devido à grande compactação do osso mandibular (Ravi, Vinay, Uday, \& Uppada, 2018). Neste contexto, o uso da articaína torna-se atraente devido sua capacidade aumentada de difusão pela membrana nervosa. Este fato permite que se alcance anestesia pulpar satisfatória na realização de infiltrações bucais, inclusive na região mandibular posterior (Haase, Reader, Nusstein, Beck, \& Drum, 2008; Malamed, 2013). Diante disto, a utilização de articaína por meio de infiltrações supraperiostais parece ser a alternativa à técnica clássica de bloqueio, garantindo maiores índices de sucesso.

O presente estudo objetivou avaliar a eficácia anestésica da técnica de infiltração supraperiostal com articaína $4 \%$ associada a epinefrina 1:100.000 em comparação com a técnica clássica de bloqueio do nervo alveolar inferior com lidocaína $2 \%$ associada ao mesmo vasoconstritor. 


\section{Metodologia}

Este estudo foi aprovado pelo Comitê de Ética em Pesquisa do Centro Universitário Católico de Vitória, ES, Brasil (CAAE: 99374818.2.0000.5070) e todos os voluntários assinaram o Termo de Consentimento Livre e Esclarecido para que pudessem participar.

Foram selecionados 60 voluntários, com características demográficas similares, de ambos os gêneros, na faixa etária de 18 a 65 anos, com indicação de restaurações classe I ou II de Black em elementos dentários posteriores mandibulares. Os procedimentos restauradores foram realizados por alunos do curso de odontologia, de maneira padrão como ocorre ao longo do curso superior. Foram incluídos indivíduos saudáveis (ASA I) e que apresentavam o elemento dentário em questão responsivo ao pulp tester. Os critérios de exclusão utilizados foram gestantes ou lactantes, indivíduos asmáticos por conta da maior propensão à alergia ao bissulfito presente nos anestésicos locais que contêm vasoconstritor adrenérgico, história de uso de medicamentos e história de alergia aos componentes do anestésico local.

Antes da realização da técnica anestésica, o elemento dentário correspondente ao procedimento restaurador foi testado com a aplicação de estímulo elétrico por meio do Pulp Tester (Odous de Deus, Minas Gerais, Brasil). Após secagem do elemento com jato de ar e realização de isolamento relativo, a ponta ativa do aparelho, contendo gel de fluoreto, foi posicionada no centro da face vestibular, de acordo com as recomendações do fabricante (Batista da Silva et al., 2010). Foram realizadas três medidas no elemento dentário e a média aritmética delas foi considerada como limiar basal da resposta pulpar. Após a realização das medidas basais, foi realizada a técnica de anestesia tópica (mucosa superficial). Antes do início da realização do procedimento restaurador, os voluntários receberam, aleatoriamente, um tubete anestésico $(1,8 \mathrm{~mL})$ em uma das seguintes técnicas: Bloqueio do nervo alveolar inferior (BNAI) realizada com lidocaína 2\% associada a epinefrina 1:100.000 (Alphacaine $^{\circledR}$ - Nova DFL) ou Técnica infiltrativa supraperiostal (INFILT) realizada com articaína 4\% associada a epinefrina 1:100.000 (Articaine ${ }^{\circledR}$ - Nova DFL). Para alocação dos participantes, uma lista de números aleatórios gerados por computador foi usada, para protocolos 1 e 2 de tratamento.

As técnicas anestésicas foram realizadas por um único operador, que não foi o pesquisador responsável pelas avaliações referentes ao sucesso da técnica realizada, de forma a garantir o cegamento da pesquisa. A anestesia pulpar foi avaliada por meio do aparelho pulp tester a cada 2 minutos após o final da injeção da solução anestésica por meio da aplicação do estímulo elétrico ao dente. O tempo de latência pulpar foi considerado como o período de tempo decorrido desde o final da injeção da solução anestésica até a ausência de percepção do estímulo elétrico na intensidade máxima emitida pelo aparelho (perda de sensibilidade). Tendo o dente alcançado anestesia (ausência de sensibilidade ao estímulo máximo do pulp tester), o mesmo passou a ser avaliado a cada 10 minutos, durante um período de 60 minutos, a fim de confirmar a eficácia da técnica anestésica utilizada, até que os 60 minutos finalizassem ou que se obtivessem duas medidas consecutivas com sensibilidade dolorosa. Considerou-se sucesso anestésico quando o elemento apresentou, pelo menos, duas medidas consecutivas sem que houvesse percepção dolorosa ao estímulo máximo do aparelho.

Para avaliação da dor à injeção, os voluntários preencheram uma escala de dor em caixa de 11 pontos, na qual marcaram a intensidade da dor sentida durante a punção e injeção do anestésico, podendo variar de zero a dez, sendo zero representativo de ausência de dor e dez representativo de maior dor possível (Jensen, Karoly, \& Braver, 1986). Após 24 horas da realização do procedimento, o voluntário foi contatado com o intuito de indagar a ocorrência de possíveis complicações pós-operatórias.

Os resultados obtidos acerca do tempo de latência e duração da anestesia foram comparados pelo teste de MannWhitney, uma vez que não apresentaram distribuição normal (teste de Shapiro-Wilks). Para a comparação sobre as frações de sucesso anestésico, foi utilizado o teste qui-quadrado. Por fim, a comparação entre os níveis de dor à punção/injeção foi feita 
pelo teste de Mann Whitney. As análises foram realizadas pelo pacote estatístico GraphPad Prism ${ }^{\circledR} 8.0$ e o nível de significância adotado foi de $5 \%(\alpha=0,05)$.

\section{Resultados}

Os resultados referentes ao tempo de latência em função da técnica anestésica utilizada estão sumarizados na Tabela 1. Ao comparar o tempo de latência dos elementos dentários que foram efetivamente anestesiados, não houve diferenças estatisticamente significantes entre as duas técnicas anestésicas ( $\mathrm{p}=0,4755$, teste de Mann-Whitney).

Tabela 1 - Tempo de latência da anestesia pulpar, em função da técnica anestésica.

\begin{tabular}{cccc}
\hline Latência (em minutos) & Dente inferior posterior & \\
& BNAI & INFILT \\
\hline 2 & 5 & 7 \\
4 & 2 & 11 \\
6 & 4 & 4 \\
8 & 1 & 1 \\
10 & 1 & 1 \\
12 & 1 & \\
14 & 1 & 6 \\
Não anestesiado & 15 & 30 \\
\hline TOTAL & 30 & \\
\hline
\end{tabular}

Fonte: Autores.

Houve diferenças estatisticamente significantes (Qui-quadrado, $\mathrm{p}=0,0149$ ) entre as técnicas anestésicas em relação ao total de dentes não anestesiados, ou seja, a técnica infiltrativa supraperiostal apresentou um sucesso anestésico significativamente maior em comparação à técnica de bloqueio do nervo alveolar inferior. $\mathrm{O}$ índice de sucesso apresentado para a técnica BNAI foi de $50 \%$, enquanto que para a técnica infiltrativa foi de $80 \%$.

Os resultados relativos à duração da anestesia pulpar estão demonstrados na Tabela 2, sendo que, àqueles elementos que não alcançaram anestesia foi atribuído o valor de zero referente à duração da anestesia pulpar. Nota-se que não houve diferenças estatisticamente significantes entre as duas técnicas anestésicas neste quesito $(\mathrm{p}=0,1381)$.

Tabela 2 - duração da anestesia pulpar, em função da técnica anestésica.

\begin{tabular}{|c|c|c|c|c|}
\hline & & \multicolumn{3}{|c|}{ Tempo de anestesia (em minutos) } \\
\hline & & & & p-valor \\
\hline & & Mediana (DIQ) & Média $( \pm \mathrm{DP})$ & \\
\hline & & & & (Mann-Whitney) \\
\hline \multirow{2}{*}{ Dente inf post } & BNAI & $0(0)$ & $22,7( \pm 29,0)$ & \multirow{2}{*}{0,1381} \\
\hline & INFILT & $30(58)$ & $31,7( \pm 25,5)$ & \\
\hline
\end{tabular}

Fonte: Autores. 
Considerando que a avaliação da duração de anestesia pulpar só foi conduzida naqueles elementos dentários que foram devidamente anestesiados, ao analisar apenas estes casos, também não foram encontradas diferenças significativas entre as técnicas anestésicas. ( $\mathrm{p}=0,1119$, teste de Mann Whitney). Neste caso, a média aritmética para a duração do efeito pulpar foi aproximadamente 52 minutos para BNAI e 43 minutos para INFILT.

A avaliação da dor após a punção anestésica foi realizada por meio de uma escala de dor em caixa de 11 pontos e os resultados estão demonstrados na Figura 1. Não houve diferenças estatisticamente significantes ( $\mathrm{p}=0,9256$, teste de MannWhitney) entre as duas técnicas anestésicas. A mediana exibida para ambas as técnicas foi 2.

Figura 1 - Sensibilidade dolorosa (escala de dor em caixa, de 11 pontos) referente à punção anestésica, relatada após bloqueio do nervo alveolar inferior (BNAI) e infiltração supraperiostal (INFILT). Barra central maior $=$ mediana; caixas $=1^{\circ}$ e $3^{\circ}$ quartis; suíças = valores máximo e mínimo. (Mann-Whitney, p=0,9256).

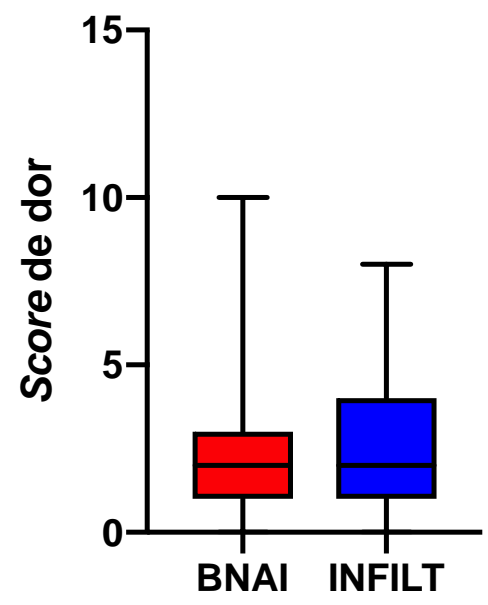

Fonte: Autores.

Por fim, sobre as complicações pós-operatórias, foram observados 11 casos em ambos os grupos, o que configura uma ocorrência de complicações em 36,7\% dos voluntários. Tais casos incluíram, principalmente, dor no local, além de edema e hematoma.

\section{Discussão}

Na presente pesquisa, o BNAI foi utilizado como controle, pois é considerada a técnica anestésica padrão para a realização de procedimentos odontológicos em dentes posteriores mandibulares. Entretanto, uma de suas principais desvantagens é a alta taxa de insucesso, principalmente por conta da dificuldade em realizar a técnica corretamente (Oleson et al., 2010; Venkat Narayanan, Gurram, Krishnan, Muthusubramanian, \& Sadesh Kannan, 2017). A infiltração bucal de anestésico local na região posterior da mandíbula não é usualmente recomendada por conta da presença de um osso denso e compacto, o que dificulta a difusão do anestésico local (Rayati, Noruziha, \& Jabbarian, 2018). Porém, com articaína, pode-se obter sucesso com técnicas infiltrativas, visto que, por conta de suas particularidades moleculares, apresenta maior lipossolubilidade e, consequentemente, maior taxa de difusão (Rathi et al., 2019).

A avaliação do sucesso anestésico foi realizada de forma objetiva, por meio do aparelho pulp tester. Alguns estudos anteriores basearam sucesso anestésico em ausência de dor durante a realização do procedimento odontológico em questão ou presença de dor leve/desconforto (Monteiro, Groppo, Haiter-Neto, Volpato, \& Almeida, 2015; Poorni, Veniashok, 
Senthilkumar, Indira, \& Ramachandran, 2011; Venkat Narayanan et al., 2017). Esta avaliação subjetiva pode não refletir precisamente a obtenção de anestesia pulpar de profundidade satisfatória. A avaliação por meio de testes elétricos da polpa já foi utilizada anteriormente e permite maior objetividade na avaliação (Corbett, Kanaa, Whitworth, \& Meechan, 2008; Srinivasan, Poorni, Nitharshika, Diana, \& Duraivel, 2017).

O tempo de latência foi medido desde a injeção anestésica até a ausência de sensibilidade dolorosa ao estímulo máximo do aparelho. Dentre os dentes que foram efetivamente anestesiados, obteve-se um tempo de latência médio de 5,7 minutos para BNAI e 4,7 minutos para INFILT, sem diferenças significativas entre os grupos. Estes resultados são ligeiramente menores que os demonstrados por Abu-Melha et al. (2018), que encontraram valores de 8,4 minutos de latência para lidocaína $2 \%$ e 7,4 minutos para infiltração de articaína 4\%. Esta diferença pode se dar pela técnica utilizada, pois, neste caso, ambas as soluções foram injetadas por infiltração bucal na região de incisivos inferiores. Entretanto, os valores do presente estudo se assemelham ao tempo de latência de 6,8 minutos para articaína em infiltração e 6,2 minutos para lidocaína em bloqueio encontrado por Corbett et al. (2008).

O sucesso anestésico foi medido em relação à proporção de dentes efetivamente anestesiados, para cada solução/técnica anestésica utilizada. Foi encontrado um sucesso de $80 \%$ para INFILT e 50\% para BNAI, o que demonstrou um índice significativamente maior para a infiltração com articaína 4\%. O fato de ter alcançado um maior índice de sucesso com o uso de articaína em infiltração bucal corrobora com o que foi apresentado por Monteiro et al. (2015), que encontrou um índice de sucesso de $40 \%$ para infiltração com articaína $4 \%$ e $10 \%$ para BNAI com lidocaína 2\%, com diferenças significativas entre os grupos. Entretanto, as taxas de sucesso dos referidos autores são menores que as do presente estudo, provavelmente por se tratar de anestesia local em elementos dentários com pulpite irreversível, enquanto no presente trabalho, não havia processo inflamatório ou infeccioso associado aos elementos dentários em análise.

Por outro lado, os índices de sucesso desta pesquisa diferem dos apresentados por Venkat Narayanan et al. (2017). Ao comparar as mesmas soluções e técnicas anestésicas, foram encontrados índices de sucesso semelhantes para os dois grupos, de 96\%. De forma semelhante, Poorni et al. (2011), ao utilizar os mesmos grupos de comparação, para tratamento de molares inferiores com pulpite, encontraram um índice de sucesso de 69,2\% para ambos os grupos em questão. Esta diferença pode, possivelmente, ser atribuída a diferenças no método para avaliação do sucesso, sendo que no presente trabalho este foi objetivo, por meio do aparelho pulp tester; enquanto que nos trabalhos referidos, se baseou apenas na possibilidade de completar o procedimento odontológico, mesmo que com presença de dor leve ou desconforto.

Apesar de Srinivasan et al. (2017) terem utilizado pulp tester para avaliação do sucesso anestésico, em uma metodologia semelhante, os resultados também apontaram semelhança estatística entre o índice para o bloqueio com lidocaína $(89,1 \%)$ e para a infiltração de articaína (82,6\%). Isto também pôde ser visto no trabalho de Corbett et al. (2008), que encontrou, em uma avaliação objetiva, um sucesso anestésico estatisticamente semelhante entre as duas técnicas, sendo de 70,4\% para infiltração com articaína e 55,6\% para bloqueio com lidocaína. Independentemente dos resultados, percebe-se uma elevada taxa de sucesso para a infiltração bucal de articaína 4\% em elementos dentários posteriores, a qual pode ser superior à técnica tradicional de bloqueio ou compatível com a mesma. De qualquer maneira, a técnica infiltrativa demonstra ser uma boa alternativa à técnica convencional.

No que diz respeito à duração do efeito anestésico, os elementos dentários foram testados por 60 minutos após a injeção anestésica. Os estudos relatados anteriormente não avaliaram o tempo de duração da anestesia pulpar, provavelmente por se tratarem de procedimentos de curta duração, o que inviabiliza a correlação entre possibilidade de completar o procedimento e duração do efeito anestésico em tecido pulpar. No presente trabalho, mesmo que o procedimento restaurador se encerrasse antes do tempo de 60 minutos, os voluntários eram mantidos sob avaliação da duração da anestesia pulpar. 
Um fator considerável em relação à realização da anestesia local é a dor à punção e injeção, que pode ser sentida em diferentes níveis pelos pacientes. No presente trabalho, a dor após a punção anestésica foi avaliada por meio da escala de 11 pontos em caixa. Esta escala é largamente utilizada e já teve seu uso validado (Jensen et al., 1986). Na presente pesquisa, foram encontrados valores medianos de 2 para ambos os grupos, o que configura a presença de dor leve (Collins, Moore, \& McQuay, 1997). Estes valores estão de acordo com os encontrados por Venkat Narayanan et al. (2017), que relataram, utilizando a mesma escala de dor, um valor médio de 2 para o grupo de infiltração com articaína e 1,16 para o grupo de bloqueio com lidocaína. Corbett et al. (2008) também encontraram resultados semelhantes, ao utilizar uma escala analógica visual, na qual a intensidade de dor é medida em centímetros, variando de zero a 10. Para a técnica infiltrativa com articaína $4 \%$ o valor médio encontrado foi de 2,24 centímetros, enquanto que para a técnica de bloqueio com lidocaína $2 \%$ foi de 2,07 centímetros.

Por fim, o índice de complicações pós-operatórias deste estudo foi de 36,7\% para cada grupo e pode-se afirmar que as complicações relatadas foram todas de baixa intensidade. Este desfecho corrobora com o trabalho de Nydegger, Nusstein, Reader, Drum, \& Beck, (2014) que, ao utilizar as mesmas soluções em elementos dentários com pulpite irreversível, apenas em técnica infiltrativa supraperiostal, também encontraram complicações pós-operatórias de baixa gravidade, sendo elas edema e sensibilidade no local da punção. Tais complicações ocorreram, para cada grupo, em uma taxa máxima de 28,3\%.

\section{Conclusão}

Com base nos resultados e dentro das limitações do estudo, conclui-se que a solução de articaína $4 \%$ com epinefrina 1:100.000, em infiltração bucal, apresenta maior índice de sucesso anestésico que a solução de lidocaína $2 \%$ com epinefrina 1:100.00, em técnica de bloqueio do nervo alveolar inferior, para procedimentos restauradores em dentes posteriores mandibulares. Além disso, uma vez alcançada anestesia, ambas as soluções apresentam tempo de latência semelhante e são capazes de alcançar uma duração de anestesia pulpar também semelhante. Por fim, devido ao baixo índice de complicações, pode-se inferir que ambas as soluções anestésicas testadas são seguras para uso na clínica odontológica.

\section{Referências}

Abu-Melha, A. S. et al. (2018). Anesthetic Efficacy of $4 \%$ Articaine Versus $2 \%$ Lidocaine in Lower Central Incisors - A Comparative Study. Dentistry. 5(4), 34-36.

Batista da Silva, C., Berto, L. A., Volpato, M. C., Ramacciato, J. C., Motta, R. H. L., Ranali, J., \& Groppo, F. C. (2010). Anesthetic Efficacy of Articaine and Lidocaine for Incisive/Mental Nerve Block. Journal of Endodontics, 36(3), 438-441. https://doi.org/10.1016/j.joen.2009.12.014

Collins, S. L., Moore, A. R., \& McQuay, H. J. (1997). The visual analogue pain intensity scale: what is moderate pain im millimeters. Pain, 72 , 95-97.

Corbett, I. P., Kanaa, M. D., Whitworth, J. M., \& Meechan, J. G. (2008). Articaine Infiltration for Anesthesia of Mandibular First Molars. Journal of Endodontics, 34(5), 514-518. https://doi.org/10.1016/j.joen.2008.02.042

Haase, A., Reader, A., Nusstein, J., Beck, M., \& Drum, M. (2008). Comparing anesthetic efficacy of articaine versus lidocaine as a supplemental buccal infiltration of the mandibular first molar after an inferior alveolar nerve block. Journal of the American Dental Association, 139(9), 1228-1235. https://doi.org/10.14219/jada.archive.2008.0338

Jensen, M. P., Karoly, P., \& Braver, S. (1986). The measurement of clinical pain intensity: a comparison of six methods. Pain, 27(1), 117-126. Retrieved from http://www.ncbi.nlm.nih.gov/pubmed/3785962

Karapinar-kazandag, M., Tanalp, J., \& Ersev, H. (2019). Effect of Premedication on the Success of Inferior Alveolar Nerve Block in Patients with Irreversible Pulpitis : A Systematic Review of the Literature. 2019.

Kaushik, M., \& Moturi, K. (2018). Local Anaesthesia and Inferior Alveolar Nerve Block - A Never Ending Saga. 5(1), 1-5. https://doi.org/10.19080/JPCR.2018.05.555652

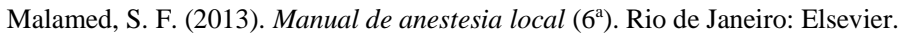

Maljaei, E., Pourkazemi, M., Ghanizadeh, M., \& Ranjbar, R. (2017). The efficacy of buccal infiltration of $4 \%$ articaine and psa injection of 2\% lidocaine on anesthesia of maxillary second molars. Iranian Endodontic Journal, 12(3), 276-281. https://doi.org/10.22037/iej.v12i3.16464 
Research, Society and Development, v. 10, n. 11, e13101119376, 2021

Monteiro, M. R. F. P., Groppo, F. C., Haiter-Neto, F., Volpato, M. C., \& Almeida, J. F. A. (2015). 4\% articaine buccal infiltration versus 2\% lidocaine inferior alveolar nerve block for emergency root canal treatment in mandibular molars with irreversible pulpits: A randomized clinical study. International Endodontic Journal, 48(2), 145-152. https://doi.org/10.1111/iej.12293

Nydegger, B., Nusstein, J., Reader, A., Drum, M., \& Beck, M. (2014). Anesthetic comparisons of 4\% concentrations of articaine, lidocaine, and prilocaine as primary buccal infiltrations of the mandibular first molar: A prospective randomized, double-blind study. Journal of Endodontics, 40(12), $1912-1916$. https://doi.org/10.1016/j.joen.2014.08.001

Oleson, M., Drum, M., Reader, A., Nusstein, J., \& Beck, M. (2010). Effect of preoperative ibuprofen on the success of the inferior alveolar nerve block in patients with irreversible pulpitis. Journal of Endodontics, 36(3), 379-382. https://doi.org/10.1016/j.joen.2009.12.030

Poorni, S., Veniashok, B., Senthilkumar, A. D., Indira, R., \& Ramachandran, S. (2011). Anesthetic efficacy of four percent articaine for pulpal anesthesia by using inferior alveolar nerve block and buccal infiltration techniques in patients with irreversible pulpitis: A prospective randomized double-blind clinical trial. Journal of Endodontics, 37(12), 1603-1607. https://doi.org/10.1016/j.joen.2011.09.009

Rathi, N. V., Khatri, A. A., Agrawal, A. G., M, S. B., Thosar, N. R., \& Deolia, S. G. (2019). Anesthetic Efficacy of Buccal Infiltration Articaine versus Lidocaine for Extraction of Primary Molar Teeth. Anesthesia Progress, 66(1), 3-7. https://doi.org/10.2344/anpr-65-04-02

Ravi, B. S., Vinay, K., Uday, M. K., \& Uppada, K. (2018). Comparison of Efficacy of Halstead , Vazirani Akinosi and Gow Gates Techniques for Mandibular Anesthesia. Journal of Maxillofacial and Oral Surgery. https://doi.org/10.1007/s12663-018-1092-5

Rayati, F., Noruziha, A., \& Jabbarian, R. (2018). Efficacy of buccal infiltration anaesthesia with articaine for extraction of mandibular molars: a clinical trial. British Journal of Oral and Maxillofacial Surgery, 56(7), 607-610. https://doi.org/10.1016/j.bjoms.2018.06.012

Srinivasan, M. R., Poorni, S., Nitharshika, Y., Diana, D., \& Duraivel, D. (2017). Articaine buccal infiltration versus lignocaine inferior alveolar block for pulpal anaesthesia in mandibular second premolars - Randomized control double blinded clinical trial. Journal of Pierre Fauchard Academy (India Section), 31(2-4), 79-83. https://doi.org/10.1016/j.jpfa.2017.03.005

Tortamano, I. P., Siviero, M., Lee, S., Sampaio, R. M., Simone, J. L., \& Rocha, R. G. (2013). Onset and duration period of pulpal anesthesia of articaine and lidocaine in inferior alveolar nerve block. Brazilian Dental Journal, 24(4), 371-374. https://doi.org/10.1590/0103-6440201302072

Venkat Narayanan, J., Gurram, P., Krishnan, R., Muthusubramanian, V., \& Sadesh Kannan, V. (2017). Infiltrative local anesthesia with articaine is equally as effective as inferior alveolar nerve block with lidocaine for the removal of erupted molars. Oral and Maxillofacial Surgery, 21(3), 295-299. https://doi.org/10.1007/s10006-017-0628-z 